\title{
Agar Assisted Embedding: Visualizing Plant Pathogen Interactions at The Root - Soil Interface
}

\section{J. Kilcrease, J. Hammond}

United States Department of Agriculture, Agricultural Research Service, U.S. National Arboretum Floral and Nursery Plants Research Unit, Beltsville, MD 20705

Soil pathogens such as viruses are wide spread and have detrimental effects on many economically important plant species. A suite of plant viruses exist that are transmitted to plant root systems through soil or by soil-borne vectors including parasitic nematodes, fungi, and protists [1]. These types of viruses and the interactions between the virus, vector (if present), and host plant are not well known and prove to be difficult to study largely in part due to problems in concurrent analysis of plant and soil [1]. Visualizing interactions of virus-soil complexes that are unstable or easily damaged presents a challenge. The key to successfully imaging the delicate root-soil interface in its native state including host, virus, and vector is maintaining sample integrity throughout the fixation - dehydration - embedment processes. Therefore the objective of this research is to; 1) utilize a liquid agar fixative medium initially to aide in the stabilization of soil / biological sample complexes in their native conformation and 2) determine how Plantago asiatica mosaic virus (PlAMV) virus particles move from infected roots into surrounding soil.

PlAMV is a member of the genus Potexvirus that affects a number of plant species, including lilies, causing mosaic or ringspot symptoms [2]. The Potexvirus group is named after one of its members potato virus $\mathrm{X}$ (PVX) and is for the most part homogenous in nature with filamentous virus particles that are 470-580 $\mathrm{nm}$ in length and approximately $12 \mathrm{~nm}$ wide with a single-stranded, positive sense RNA genome [2]. While PlAMV was first discovered in the Russian Far East, it was reported in 2010 in the Netherlands to reduce lily cut-flower production by almost $80 \%$ [3]. More recently the virus has been found on Asiatic and Oriental lilies imported to the United States and if not controlled could significantly impact domestic cut flower lily production [4]. A vector has not been found, but it has been reported that the virus can spread via mechanical transmission by root-to-root contact and through soil at the root-soil interface [5].

Transmission electron microscopy (TEM) methods were used to investigate the effects of PlAMV in root-soil samples of Nicotiana benthamiana and Asiatic / Oriental lilies (Lilium sp.). These findings demonstrate the architecture / interactions of the virus in the root and at the root-soil interface utilizing an agar assisted fixation protocol to stabilize the natural conformation of soil in direct contact with roots. Lilies with known PIAMV infection along with manually inoculated $N$. benthamiana plants were used and root balls with soil intact extracted from pots. A liquid solution of $3 \%$ agar in $0.05 \mathrm{M}$ phosphate buffer was prepared with glutaraldehyde added (after heating) to a final concentration of $3 \%$. The solution was then dripped onto small feeder roots surrounded by an intact soil matrix. The agar fixative solution was allowed to solidify and the agar embedded section carefully removed from the root ball. This extracted area was placed into a vessel containing the same liquid agar fixative solution and held at $4{ }^{\circ} \mathrm{C}$ overnight. Agar embedded root-soil samples (Fig. 2) were further trimmed, stained with osmium tetraoxide, and embedded into LX-112 resin. Ultrathin sections were obtained utilizing a DiATOME Ultra 45 knife and imaged with a JEOL JEM-100CX transmission electron microscope at 80kV. Phosphotungustic acid (PTA) was utilized to negatively stain leaf dips and root grinds from infected plants. 
Successful confirmation of PlAMV infection in Lilium sp. and N. bentamiama was determined via leaf dips (Fig. 3), negatively stained soil preparations, and PCR (data not shown). The root-soil embedded tissue using the agar assisted procedure resulted in composite samples containing both root and soil entities with undisturbed architecture (Fig. 1 A-C). To date we have not detected virus extrusion or free PIAMV particles outside of the root wall, however this method of utilizing a liquid agar fixative solution has been demonstrated to be very useful in the TEM visualization of the rootsoil interface. It could also be used in instances of soil-borne vector and/or other pathogen interaction with roots as well as a stabilizer in pre-embedment processing for bacterial colony matrices, fungal networks, and other unstable targets. Further studies of viral interactions and architecture in soil-borne scenarios are likely to reveal more information about host-pathogen-vector etiology [6].

\section{References:}

[1] A. G. Roberts, eLS - John Wiley \& Sons Ltd, Chichester, Plant Viruses: Soil-borne (2014).

[2] K. Komatsu, et. al. Arch. Virol. 153 (2008) 193-198

[3] Anonymous. https://www.vwa.nl/txmpub/files/?p_file_id=2001424, accessed Feb. 10, 2015.

[4] J. Hammond, Plant Disease 99 (2015) 292.

[5] C.G.M. Conijn, Acta Hort 1027 (2014) 213-229.

[6] Acknowledgements: Funding by Oak Ridge Institute of Science and Engineering \& USDA
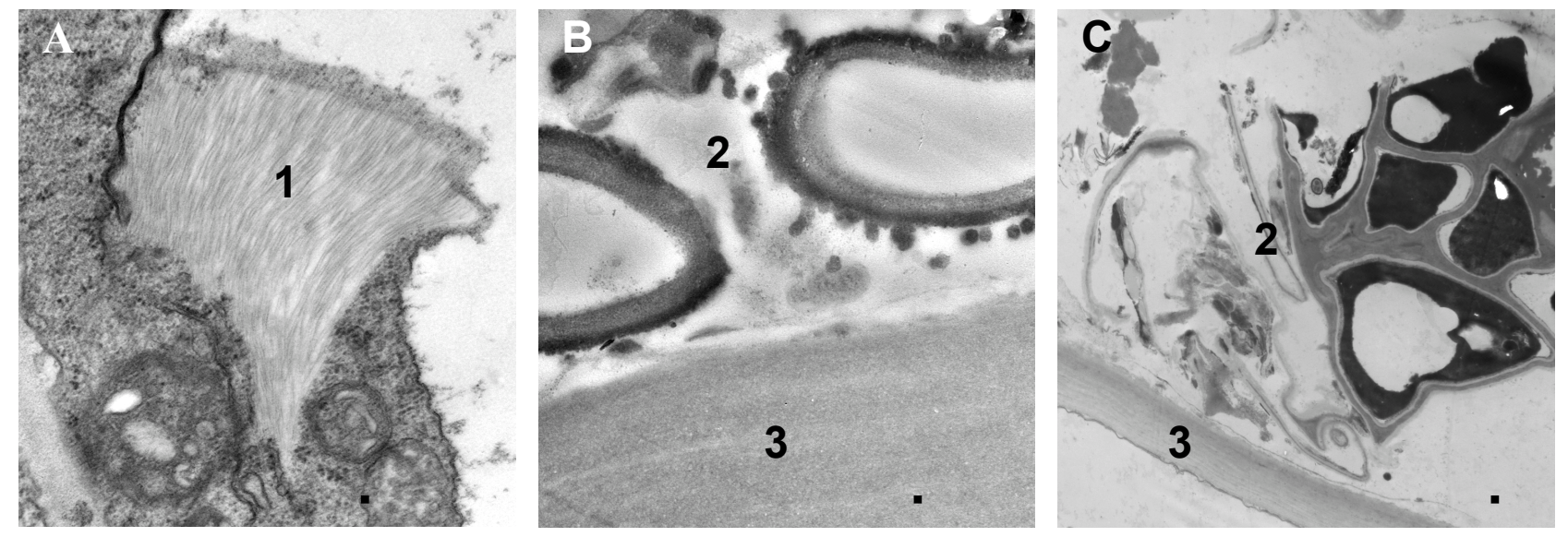

Fig. 1. TEM images of root and the root - soil interface in plants infected with Plantago asiatica mosaic virus. A: $N$. benthamiana root with PIAMV virus accumulation (1) near cell wall. B, C: Root - soil interface demonstrating undisturbed soil particulate (2) outside of root epidermal wall (3) of $N$. benthiama and Lilium sp. respectively. Scale bars $=500 \mathrm{~nm}$.

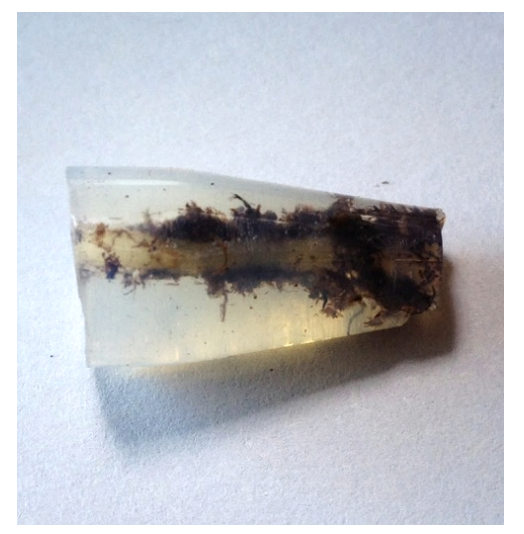

Fig. 2. (Left) Root-soil matrix of lily in native conformation embedded in agar - fixative solution prior to dehydration.

Fig. 3. (Right) PlAMV virus particles from infected $N$. benthamiama leaf negatively stained with PTA. Scale bar = $500 \mathrm{~nm}$.

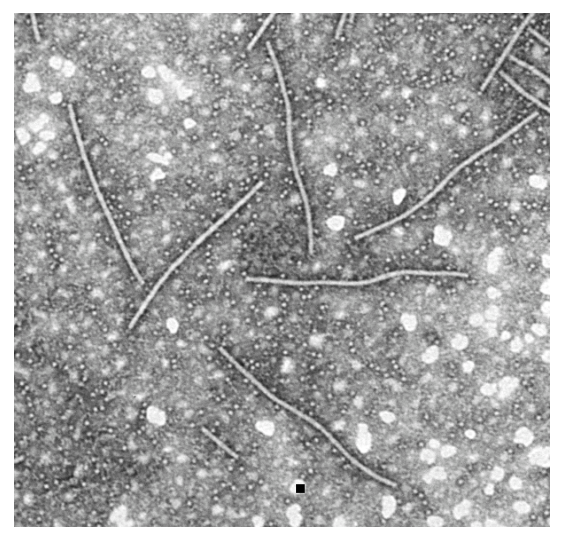

